\title{
FAST CHARGE COLLECTION IN GaAs MESFETs
}

\author{
Dale McMorrow, Alvin R. Knudson and Arthur B. Campbell \\ Naval Research Laboratory \\ Washington, D.C. 20375
}

\begin{abstract}
Time-resolved charge collection measurements on $1 \mu \mathrm{m}$ gate length digital GaAs MESFETs with a variety of energetic ions and picosecond laser pulses exhibit risetimes as short as 25 ps with pulsewidths of $\sim 35$ ps. Evidence is presented for the presence of three distincttimescale charge collection processes, with time constants ranging from less than 25 ps to $>1 \mu \mathrm{s}$. The effects of radiation damage on the chargecollection transients are presented, and the use of above band-gap picosecond laser excitation is demonstrated as a viable alternative to ion excitation for characterization of the dynamical response of high-frequency, radiation-sensitive devices to rapid charge deposition.
\end{abstract}

\section{Introduction}

Energetic ions interact with semiconductor devices to produce ionization tracks that consist of dense plasmas of free carriers. These rapidly thermalize with the lattice ( $<2$ ps for $300 \mathrm{~K}$ $\mathrm{GaAs}$ ) and, under the influence of drift and diffusion, are collected at the various circuit nodes. Earlier measurements of charge collection on the picosecond time scale dealt with largearea silicon and GaAs diodes [1], which exhibited ion-induced current pulses exhibiting full-width-at-half-maximum pulsewidths on the order of one-hundred to several hundred picoseconds.

In this paper we report measurements of the ion-induced and picosecond-laser-induced current transients produced in high-speed, digital gallium arsenide metal-semiconductor fieldeffect-transistors (MESFETs). Charge-collection transients exhibiting risetimes on the order of $25 \mathrm{ps}$, with full-width-at-half-maximum pulsewidths of $\sim 35 \mathrm{ps}$, are observed in this work. These data reveal the presence of at least two, and possibly three, dynamically-distinct contributions to the charge collection transients for both laser and ion excitation. In addition, the results presented illustrate the sensitivity of the current transients produced in these high-speed $\mathrm{GaAs}$ devices to the effects of radiation damage. The combination of radiation-damage effects, coupled with the bandwidth limitations of the surrounding circuit and/or packaging, can give rise to a lack of dependence of the measured transients on the stopping power of the incident ion. Radiation damage is particularly rapid for the heavier ions of this study (B, O, and $\mathrm{Si}$ ). For excitation with ${ }^{4} \mathrm{He}$, however, damage is sufficiently slow that charge collection transients for nominally undamaged devices are obtained.

We show that charge-collection measurements with above band-gap picosecond laser irradiation reproduce all of the qualitative features of ionic excitation on undamaged devices. Therefore, it is suggested that laser excitation can be used as a viable alternative to heavy ions for characterization of the dynamical response of high-frequency semiconductor devices to rapid charge deposition, free from the effects of radiation damage.

These measurements are important not only for a basic understanding of the physical phenomena associated with ion/solid interactions, but also for an understanding of SEU phenomena in modern high-speed digital circuits. GaAs technology offers high-speed, totaldose hardness, and latch-up resistant digital circuit elements. Single-event upsets, however, present significant reliability problems because of the low capacitance and enhanced charge collection of these devices [2-4]. To predict the response of complex circuits to ion strikes on a given element requires knowledge of the current pulse produced by the ion, the response of the transistor element, as well as a knowledge of the circuit response. Simulation codes such as SPICE require a mathematical input function to approximate the response of a given circuit element to an ion strike. When the circuit response time is of the order of the input pulse duration, the prediction of SEU sensitivity can depend critically on the amplitude, shape, and duration of the pulse. Thus it is important to know not only the total amount of charge deposited in a circuit element, but also the time dependence of the current pulse. Only then is it possible to propagate that pulse in a circuit simulation and make reliable predictions of SEU sensitivity.

\section{Experimental}

The current transients produced by the ion strikes and laser pulses were measured with a HYPRES PSP-750 high-bandwidth, superconducting sampling oscilloscope. The unique ad-

U.S. Government work not protected by U.S. copyright

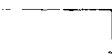




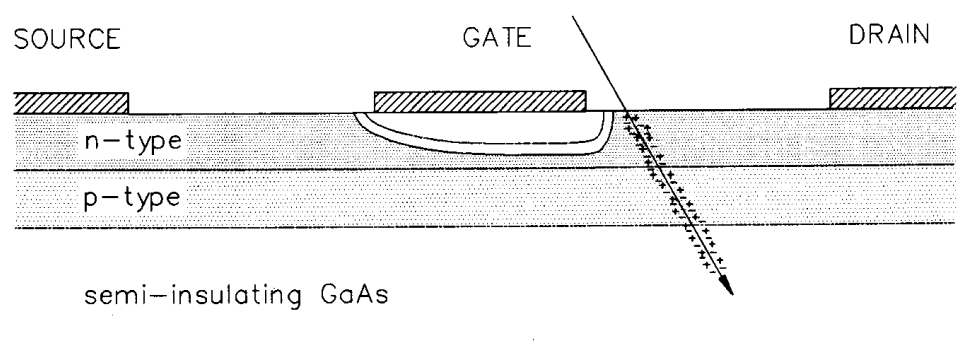

Figure 1. Schematic diagram of an ion track in a $1 \mu \mathrm{m}$ gate length depletion-mode GaAs MESFET typical of those used in this study.

vantage of this instrument for the present experiments is its built-in, effectively dispersion-free, lossless, superconducting delay line that allows the sampling of randomly arriving transients, while maintaining its full $70 \mathrm{GHz}$ bandwidth. For biasing purposes an HP $50 \mathrm{GHz}$ bias tee was inserted in the line betwen the semi-rigid cable and the front end of the PSP-750. This tee blocks the DC drain bias from the front end of the signal processor, while passing the highfrequency $\mathrm{AC}$ components.

Measurements were performed on both depletion and enhancement mode GaAs test structures fabricated by Vitesse Semiconductor as standard electrical test structures for paramet ric analysis of their enhancement/depletion process. A schematic diagram of a $1 \mu \mathrm{m}$ gate length depletion-mode device is given in figure 1. The devices were fabricated by ion implantation into un-compensated, semi-insulating material with a $\mathrm{p}^{-}$implant for threshold control, $\mathrm{n}^{+}$ implants for source, drain, and channel, and a Schottky barrier gate. The active depth of these devices is approximately $0.2 \mu \mathrm{m}$, with gate lengths ranging from $1 \mu \mathrm{m}$ to $30 \mu \mathrm{m}$. The pinch off voltages for the depletion-mode devices are typically $-1 \mathrm{~V}$. The test chips were wire bonded to $50 \Omega$ microstrip lines mounted in highfrequency $(50 \mathrm{GHz})$ microwave packages. The packages were mounted in a vacuum chamber on the accelerator beam line, and connected to the measurement system with a semi-rigid coaxial cable fed-through the vacuum wall.

The NRL ion microbeam facility was used for this work, allowing for positioning of an $\sim 4 \mu \mathrm{m}$ diameter beam on the MESFET test structures with $1 \mu \mathrm{m}$ accuracy. This facility and the associated experimental procedures have been described in detail elsewhere [3,5]. The largest amplitude (typically $\sim 5 \mathrm{mV}$ ) drain transients are observed for ion strikes in the high field region between the gate and drain with the devices pinched off. All signals reported correspond to these conditions.

Measurements were also performed using above band-gap, pulsed laser excitation at 527 $\mathrm{nm}$ and $800 \mathrm{~nm}$. These measurements permit characterization of the dynamical properties of the device without the effects of radiation damage. Laser pulses of $\sim 10 \mathrm{ps}$ duration focussed to $\sim 3 \mu \mathrm{m}$ were typical.

\section{Results and Discussion}

Figure 2 gives representative current transients measured at the drain of the $30 \mu \mathrm{m} \times 60$ $\mu \mathrm{m}$ depletion mode device for four different ions $\left(2.9 \mathrm{MeV}^{4} \mathrm{He}, 7.8 \mathrm{MeV}{ }^{10} \mathrm{~B}, 15.7 \mathrm{MeV}^{16} \mathrm{O}\right.$, and $17.2 \mathrm{MeV}^{28} \mathrm{Si}$ ). These curves represent some of the first such measurements of ion-induced transients reported for a high-speed digital $\mathrm{GaAs}$ device. For each of these curves the gate was biased at $-1.2 \mathrm{~V}$ (at pinch-off), the drain at 3.0 $V$, with the source grounded. Each curve of figure 2 was obtained after some degree of

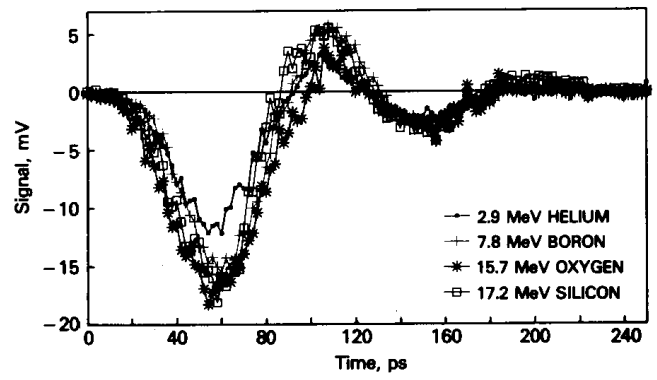

Figure 2. Representative drain current transients produced in a $30 \mu \mathrm{m} \times 60 \mu \mathrm{m}$ depletion-mode GaAs MESFET as a function of the incident ion. Similar transients are observed for the shorter gate length devices. For this series of curves the gate was biased at $-1.2 \mathrm{~V}$ and the drain at $3.0 \mathrm{~V}$. These transients were obtained after a significant amount of radiation exposure by each of the ions indicated, and exhibit the characteristics of a radiation damaged device (see text). 
radiation exposure of the device (vide infra). The data of figure 2 correspond to ion strikes in the high-field region between the gate and drain (cf., figure 1), where the largest signal amplitudes are typically observed.

The ion tracks produced by different ions can be quite different [4], with the different ions of this study exhibiting dramatically different stopping powers ( $\mathrm{He} \sim 290 ; \mathrm{B} \sim 1230 ; \mathrm{O} \sim 2350$; $\mathrm{Si} \sim 4640 \mathrm{KeV} / \mu \mathrm{m})$, giving rise to significant differences in the radial extent of the charge column. The total penetration depths of the different ions are similar ( $\mathrm{He} \sim 8.8 \mu \mathrm{m} ; \mathrm{B} \sim 6.9$ $\mu \mathrm{m} ; \mathrm{O} \sim 7.5 \mu \mathrm{m} ; \mathrm{Si} \sim 5.6 \mu \mathrm{m})$. It is intuitively expected that the physical differences in charge deposition (track structure) will translate into differences in the charge collection dynamics. Therefore, it is somewhat surprising that the dynamics observed for the four very different ions are so similar, with each exhibiting a pulse risetime of $\sim 25 \mathrm{ps}$ and a pulse width of $\sim 35 \mathrm{ps}$. The results of an earlier study [1] performed on large area silicon diodes (which showed dramatic pulse shape variations as a function of the incident ion) are consistent with this expectation. In those measurements [1] the transients produced by the heavier ions were significantly slower than those of the lighter ions. That same study, however, presented limited data on the transients produced in GaAs diodes, with no dramatic dependence of the dynamics on the incident ion being observed. We have measured the ion-induced transients for several $\mathrm{GaAs}$ MESFETs of different dimensions (ranging from $1 \mu \mathrm{m} \times 10 \mu \mathrm{m}$ to $1 \mu \mathrm{m} \times 50 \mu \mathrm{m}$ to $30 \mu \mathrm{m} \times 60$ $\mu \mathrm{m}$ ), each of which (with the exception of the ${ }^{4} \mathrm{He}$ data discussed below) exhibits dynamics similar to those of figure 2. It is demonstrated below that this unexpected lack of dependence on the nature of the incident ion can be accounted for in terms of radiation damage effects.

Charge collection measurements with energetic ions are known to be sensitive indicators of radiation damage [7]. High-speed, small area GaAs devices are particularly sensitive to such effects $[8,9]$. The data of figure 3 illustrate the effects of permanent radiation damage (displacement damage) by $2.9 \mathrm{MeV}{ }^{4} \mathrm{He}$ ions on the charge collection dynamics of a $1.2 \mu \mathrm{m} \times 50 \mu \mathrm{m}$ depletion mode device. The two curves correspond to the first and last of a series of four sucessive runs, with each run exposing the device for approximately five minutes (corresponding to approximately 100,000 ion strikes).

Evident in the first run (lower curve, which represents a nominally undamaged device) is an

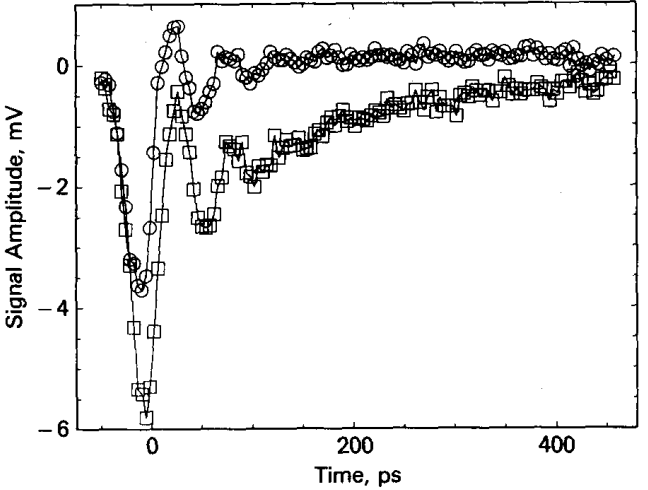

Figure 3. Current transients detected at the drain of a $1.2 \mu \mathrm{m} \times 50 \mu \mathrm{m}$ depletion-mode GaAs MESFET as a function of exposure to a $2.9 \mathrm{MeV}{ }^{4} \mathrm{He}$ ion beam $\left(\mathrm{V}_{\mathrm{G}}=-1.0 \mathrm{~V} ; \mathrm{V}_{\mathrm{D}}=4.0 \mathrm{~V}\right)$. The data presented correspond to the first (lower curve, squares) and last (upper curve, circles) of four sucessive data acquisition runs. Note the exponentially decaying component that is evident in the first curve, but is no longer present following radiation exposure.

exponential tail exhibiting a time constant of $\sim 150$ ps. The upper curve of figure 3 reveals the effects of radiation damage on the charge collection transient, with the exponential tail no longer being observable. The decay time of this component is observed to decrease with increasing radiation exposure, presumably because of the increased concentration of recombination sites introduced by the ion, causing a reduced carrier lifetime. We note that the use of ion implantation to reduce carrier lifetimes is a common procedure in the fabrication of ultrafast opto-electronic devices $[10,11]$.

It is particularly instructive to compare the data of figures 2 and 3 . The transients of figure 2 exhibit the same dynamical characteristics as the radiation damaged transient of figure 3 (upper curve). Therefore, it may be concluded that the curves of figure 2 are representative of a radiation damaged device. In fact, for the ions investigated with a stopping power greater than that of ${ }^{4} \mathrm{He}$, the rate of radiation damage is sufficiently rapid that it is difficult to collect a even single transient that does not show the effects of radiation damage. This result illustrates an intrinsic liability of sampling techniques for the measurement of ion-induced current transients. We note that, with the technology that is currently available, sampling techniques are the only method available for measuring the transients produced by randomly arriving ions with the necessary bandwidth. One 


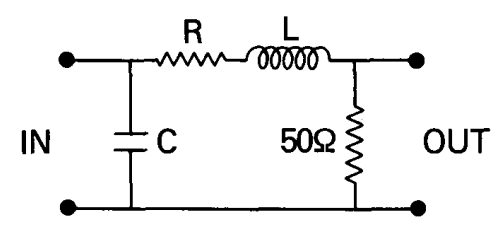

Figure 4. RCL circuit used to model the chargecollection dynamics.

possibility for minimizing radiation damage effects involves heating the device, as is suggested in reference [9]. This procedure is currently under investigation.

A fundamental question associated with the interpretation of the transient data is the origin of the pronounced oscillations. These oscillations, which typically exhibit a frequency $\omega / 2 \pi$ of $\sim 11 \mathrm{GHz}$, appear (to some extent) in all of the data collected to date, both before and after radiation damage. While there are a number of potential causes for the appearance of such oscillatory structure, including Gunn oscillations and reflections from transmission line discontinuities, simple considerations suggest that in the present case they arise from the "ringing" associated with the RLC circuit produced by the intrinsic capacitance and resistance of the device coupled to the inductance of the source and drain bond wires. The MESFETs of this study were wire-bonded to the microstrip lines with rather long $(\sim 1.5 \mathrm{~mm})$ bond wires, with the combined inductance created by the source and drain bond wires approximated to be on the order of $1.5 \mathrm{nH}$. Using the simple RLC circuit model given in figure 4 , we have been able to accurately reproduce the measured transients using an input waveform consisting of a $40 \mathrm{ps}$ rise and a $150 \mathrm{ps}$ exponential decay with an effective inductance of $3 \mathrm{nH}$, consistent with the above considerations (values of $R=50 \Omega$ and $\mathrm{C}=50 \mathrm{fF}$ were used; the longer time-constant relaxation discussed below was neglected). To further test this hypothesis, we have been able to modify the oscillation frequency by changing the bond wire lengths. Using the simple RLC circuit description, the charge collection transient produced for a device after lengthening the drain bond wire can be reproduced by increasing the value of the inductance while holding all other parameters fixed.

At this point it is possible to discuss the data of figure 2 in more detail. The lack of dependence of the observed transients on the nature of the incident ions ions can be accounted for in terms of a combination of radiationdamage and bandwidth-limiting packaging effects. The considerations of the previous paragraphs suggest that the inductances associated with the packaging of the high-frequency devices have a bandwidth-limiting effect on the measured transients, while the effects of radiation damage are to decrease the carrier lifetimes. As the carrier lifetimes become short compared to $1 / \omega_{e q}$, where $\omega_{e q}$ is the characteristic frequency of the device/package equivalent RLC circuit, the measured transients will be dominated by the RLC circuit response. This progression is evident in the data of figure 3 . In the current case, $\omega_{e q}$ is determined to a large extent by bond-wire inductances. Devices currently are being fabricated to minimize and/or eliminate such effects.

To gain added insight into the dynamics of the charge collection process, as well as to better characterize the intrinsic properties of the devices without the complications introduced by radiation damage effects, we have initiated a parallel program to investigate the current transients created by ultrashort laser pulses. The laser technique possesses an inherently higher temporal resolution, is much less expensive, more convienient, and does not produce the cumulative radiation damage that is characteristic of ion bombardment. It previously has been demonstrated [12] that the use of focussed picosecond laser pulses providing above bandgap excitation is a viable method for locating SEU sensitive areas in complex IC's.

Figure 5 gives a representative drain

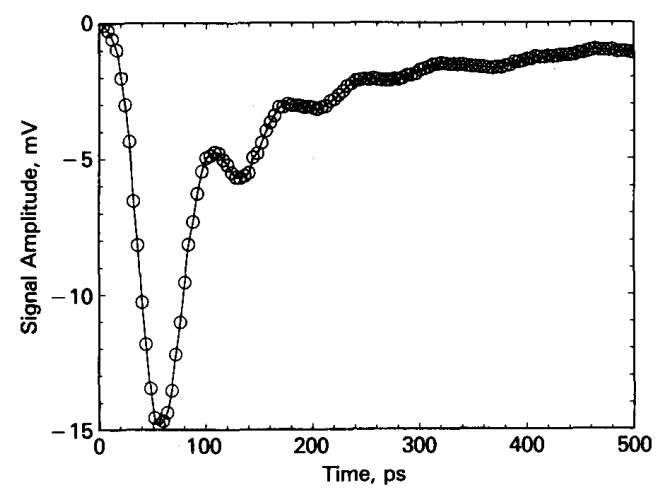

Figure 5. Current transient detected at the drain of an undamaged $1.2 \mu \mathrm{m} \times 50 \mu \mathrm{m}$ depletion-mode GaAs MESFET following excitation with a $10 \mathrm{ps}, 800 \mathrm{~nm}$ laser pulse $\left(\mathrm{V}_{\mathrm{G}}=-1.5 \mathrm{~V} ; \mathrm{V}_{\mathrm{D}}=5.0 \mathrm{~V}\right)$. Evident are all of the features present in the transient produced by ${ }^{4} \mathrm{He}$ excitation of an undamaged device ( $c f$., figure 3 ). The laser-pulse repetition rate was $400 \mathrm{MHz}$. 


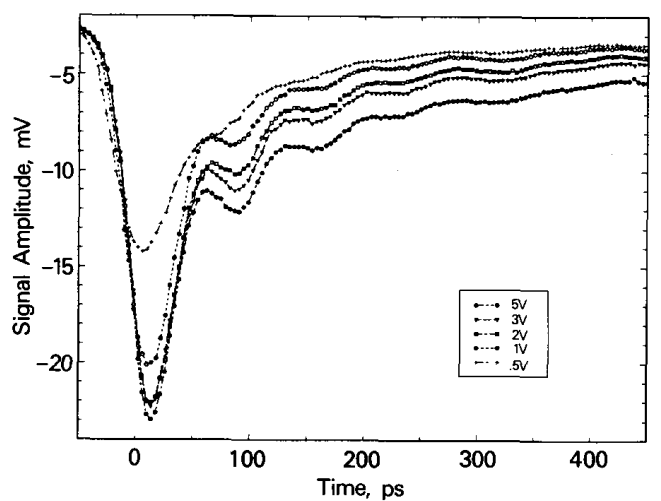

Figure 6. Current transients detected at the drain of an undamaged $1.2 \mu \mathrm{m} \times 50 \mu \mathrm{m}$ depletion-mode GaAs MESFET following excitation with a $10 \mathrm{ps}, 800 \mathrm{~nm}$ laser pulse as a function of drain bias. Note the low frequency contribution that increases in amplitude with increasing drain bias. The laser-pulse repetition rate was $400 \mathrm{MHz}$.

current transient produced for $\sim 10 \mathrm{ps}, 800 \mathrm{~nm}$ excitation of a $1.2 \mu \mathrm{m} \times 50 \mu \mathrm{m}$ depletion mode device. The observed dynamics are both qualitatively and quantatively very similar to those for ${ }^{4} \mathrm{He}$ excitation of the undamaged device ( $c f$., figure 3 , lower curve), illustrating the feasibility of simulating ion-induced charge collection dynamics with ultrashort optical pulses. The correspondence of the laser data of figure 5 with the ion data of figure 3 (lower) reenforces the assertion that the data of figure 3 (lower) is, in fact, a characteristic ion-induced charge collection transient of an undamaged device. Also evident in the laser data of figure 5 is the dramatically improved signal-to-noise ratio over that of the ion experiment, which is a consequence of the laser stability and the increased signal averaging capabilities that are possible when radiation damage is not a problem. In what follows we take advantage of the unique properties of laser excitation to illustrate some of the intrinsic dynamical properties of the GaAs devices of this study.

Accepting that picosecond laser excitation can reproduce the essential characteristics of the ion-induced charge collection transients, it is possible to utilize the optical technique to examine the the GaAs devices in greater detail, without the potentially damaging effects of ionizing radiation. Figure 6 illustrates the dependence of the detected drain current transients in a $1.2 \mu \mathrm{m} \times 50 \mu \mathrm{m}$ depletion mode device on drain voltage. The gate bias is $-1.5 \mathrm{~V}$ for each curve (device pinched off). The data is shown for 10 ps, $800 \mathrm{~nm}$ pulsed laser excitation; similar results are observed for ${ }^{4} \mathrm{He}$ ionic excitation.

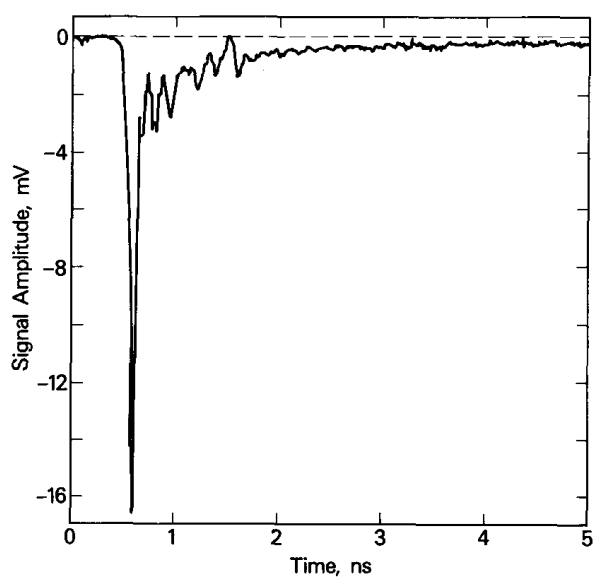

Figure 7. Current transient detected at the drain of an undamaged $1.2 \mu \mathrm{m} \times 50 \mu \mathrm{m}$ depletion-mode GaAs MESFET following excitation with a $10 \mathrm{ps}, 527 \mathrm{~nm}$ laser pulse. The $5 \mathrm{~ns}$ temporal window of this data reveals clearly the presence of a slow $(\tau \sim 1 \mu \mathrm{s})$ charge collection mechanism. The laser repetition rate was $400 \mathrm{~Hz}$.

Several features are evident in this data set: $i$ ) for $0.5 \mathrm{~V}$ drain bias the transient exhibits a slower response than at higher bias conditions, with less ringing being evident; $i$ ) for drain voltages $>1 \mathrm{~V}$ the observed transients do not exhibit a dramatic dependence on bias; but iii) the data reveals evidence for a long-lived current transient $(\tau>1 \mathrm{~ns})$ that increases in amplitude with increasing drain bias.

Thus, for drain voltages greater than $1 \mathrm{~V}$, the transients appear to consist of three dynamical regions: $i$ ) an initial, rapidly decaying contribution followed by ii) an intermediate relaxation exhibiting a time constant of $\sim 150$ ps, and iii) the slower transient noted above. The presence of a fast relaxation component $(<25 \mathrm{ps})$ is consistent with a rapid sweep-out (drift) of the carriers from the active region of the device, and is more prominent in some data sets ( $c f$. , figure 7 , vide infra). A definitive observation and characterization of a fast relaxation component, however, will require a minimization of the effects of the bandwidthlimiting packaging effects discussed above. The observation of an intermediate time constant relaxation is consistent with charge collection via the slower diffusion process, presumably associated with carriers in the low-field regions of the device.

The presence of a slow charge collection transient is evident in the data of figures 3,5 , and 6 , but this signal contribution is revealed more clearly in figure 7. Figure 7 shows a laser- 
induced drain current transient detected over a $5 \mathrm{~ns}$ time widow, produced by $\sim 10 \mathrm{ps}$ laser excitation at $527 \mathrm{~nm}$, and detected by a Tektronix 11801 sampling oscilliscope. The 1.2 $\mu \mathrm{m} \times 50 \mu \mathrm{m}$ depletion mode device was biased with a gate voltage of $-0.92 \mathrm{~V}$ and a drain voltage of $4.0 \mathrm{~V}$. This data illustrates clearly the three different relaxation regions noted in the previous paragraph. In particular, the long-lived component is seen to be almost constant on the time scale plotted. The presence of a slow charge collection mechanism is also evident in data collected as a function of the laser pulse repetition rate. At pulse repetition rates of greater than $800 \mathrm{KHz}$, the charge collection waveform becomes distorted, consistent with a device charging process. At higher repetition rates $\geq 1$ $\mathrm{MHz}$ ( $\leq 1 \mu \mathrm{s}$ pulse separation) the HYPRES input rapidly becomes saturated, and no waveform can be detected. Based on these observations we estimate a relaxation time on the order of or greater than $1 \mu \mathrm{s}$ for this transient. Recognizing that this is the characteristic time scale for the relaxation of various deep-level traps that are known to be prevalent in GaAs $[13,14]$, we tentatively assign the slow $(\sim 1 \mu \mathrm{s})$ transient to charge collection of carriers emitted from such traps. Further work should identify this contribution more precisely. We note that in the ion studies both the intermediate lifetime (ii) and the slower component (iii) decrease in amplitude with increasing radiation damage.

\section{Conclusions}

We have presented some of the first measurements of the current transients produced by energetic ions in high-speed digital GaAs MESFET devices. Ionic excitation of the GaAs MESFETs of this study produces current pulses with rise times on the order of $25 \mathrm{ps}$ and full-width-at-half-maximum durations as short as $35 \mathrm{ps}$.

The transients produced by ${ }^{4} \mathrm{He}$ and picosecond laser irradiation appear to reveal the existence of three distinct-timescale charge collection processes: $i$ ) a rapidly decaying component that is faster than the temporal resolution of the detection system that is consistent with rapid sweep out of the carriers from the active region of the device; ii) an intermediate lifetime component $(\tau \sim 150 \mathrm{ps})$ that is presumably associated with the diffusion of carriers excited outside the high-field depletion region of the device; and iii) a slow component exhibiting a lifetime on the order of $1 \mu \mathrm{s}$ that is consistent with charge collection from deep traps, known to be present in GaAs.

The effects of radiation damage on the picosecond charge collection processes of $\mathrm{GaAs}$ MESFETs are are presented for the first time. It is revealed that for all ions investigated with a stopping power greater than that of ${ }^{4} \mathrm{He}$, the effects of radiation damage are quite severe. This result represents an intrinsic liability of the sampling techniques that are necessary to detect transients produced by the randomly arriving ions. It should be emphasized, however, that radiation damage effects are sufficiently slow for $2.9 \mathrm{MeV}{ }^{4} \mathrm{He}$ excitation that charge-collection transients characteristic of the undamaged devices are obtained.

The effect of radiation exposure is to increase the decay rate of the various transient responses. This observation is consistent with the production of recombination centers that reduce the carrier lifetimes. For the heavier ions, a surprising lack of dependence on the nature of the different ions is observed ( $c f$. , figure 2). This result can be explained in terms of a combination of radiation damage effects and stray inductance effects: the radiation damage reduces the carrier lifetimes to the extent that the observed transients are determined by the bandwidth limitations of the microwave packages used. In future work such packaging effects will be minimized.

Preliminary measurements with pulsed, picosecond laser irradiation suggest that laser excitation is a viable alternative to the use of heavy ions for characterization of the dynamical response of high-frequency semiconductor devices to rapid charge deposition, free from the effects of radiation damage. While a detailed comparison of ion and laser irradiation is yet to be performed, the optical transients presented here are both qualitatively and quantatively comparable to those produced by ${ }^{4} \mathrm{He}$ excitation of undamaged devices. The superior signal-tonoise ratio of the laser data gives rise to a more precise picture of the intrinsic dynamics of the device.

\section{Acknowledgements}

The authors acknowledge useful discussions with W.J. Stapor and T.R. Weatherford. The picosecond laser experiments were performed in the laboratories of Prof. C.H. Lee at the University of Maryland, College Park, and Prof. $R$. Chen at the University of Maryland, Baltimore County. 


\section{References}

1. R.Wagner, N. Bordes, J. Bradley, C. Maggiore, A. Knudson, A. Campbell, "Alpha-, Boron-, Silicon-, and Iron-Ion-Induced Current Transients in Low-capacitance Silicon and GaAs Diodes", IEEE Trans. Nuc. Sci. NS-35, 1578-1584 (1988).

2. P.J. McNulty, W. Abdel-Kader, A.B. Campbell, A.R. Knudson, P. Shapiro, R. Eisen and S. Roosild, "Charge Collection in GaAs Test Structures", IEEE Trans. Nuc. Sci. NS-31, 1128-1131 (1984).

3. A. Campbell, A. Knudson, D. McMorrow, W. Anderson, J. Roussos, S. Espy, S. Buchner, K. Kang, D. Kerns, and S. Kerns, "Ion Induced Charge Collection in GaAs MESFETs", IEEE Trans. Nuc. Sci. NS-36, 2292-2299 (1989).

4. T.R. Weatherford, J.R. Hauser, and S.E. Kerns, "Basic Mechanisms in GaAs SEU Response", J. Rad. Effects, Res. \& Engrg. 6, 56-62 (1988).

5. A.R. Knudson and A.B. Campbell, "Use of an Ion Microbeam to Study Single Event Upsets in Microcircuits”, IEEE Trans. Nuc. Sci. NS-28, 4017-4021 (1981).

6. W.J. Stapor and P.T. McDonald, "Pratical Approach to Ion Track Energy Distribution", J. Appl. Phys. 64, 4430 (1988).

7. M.A. Xaspos, A.B. Campbell, A.R. Knudson, W.J. Stapor, P. Shapiro, T. Palmer, P.T. McDonald, and S.L. Swickert, "Charge Collection Efficiency Related to Damage in MOS Capacitors", IEEE Trans. Nuc. Sci.
NS-34, 1214-1219 (1987).

8. W.T. Anderson Jr., A.B. Campbell, A.R. Knudson, A. Christou, and B.R. Wilkins, "Degradation in GaAs FETs Resulting from Alpha Particle Irratiation", IEEE Trans. Nuc. Sci. NS-31, 1124-1127 (1984).

9. R.A. Brown and J.J. Williams, "Ion Beam Damage Phenomena in GaAs", in Proceedings of the 6th Australian Conference on Nuclear Techniques of Analysis, Lucas Heights, N.S. Wales, November, 1989, pp. 80-82.

10. P.R. Smith, D.H. Auston, A.M. Johnson, and W.M. Augustyniak, "Picosecond Photoconcudtivity in Radiation-Damaged Silicon-on-Sapphire Films", Appl. Phys. Lett. 38, 47-50 (1981).

11. see also: Picosecond Optoelectronic Devices, edited by Chi H. Lee (Academic Press, New York, 1984).

12. S. Buchner, D. Wilson, K Kang, D. Gill, J. Mazer, W. Raburn, A. Campbell, and A. Knudson, "Laser Simulation of Single Event Upsets", IEEE Trans. Nuc. Sci. NS-34, 1228-1233 (1987).

13. G.M. Martin, A. Mitonneau, and A. Mircea, "Electron Traps in Bulk and Epitazial GaAs Crystals", Electron. Lett. 13, 191-193 (1977).

14. S. Sriram and M.B. Das, "Characterization of Electron Traps in Ion-Implanted GaAs MESFET's on Undoped and Cr-Doped LEC Semi-Insulating Substrates", IEEE Trans. Elect. Dev. ED-30, 586-592 (1983). 